\title{
NON-CUT, SHORE AND NON-BLOCK POINTS IN CONTINUA
}

\author{
Jozef Bobok, Pavel Pyrih and Benjamin Vejnar
}

Czech Technical University in Prague and Charles University in Prague,

Czech Republic

\begin{abstract}
In a nondegenerate continuum we study the set of noncut points. We show that it can be stratified by inclusion into six natural subsets (containing also non-block and shore points). Among other results we show that every nondegenerate continuum contains at least two nonblock points. Our investigation is further focused on both the classes of arc-like and circle-like continua.
\end{abstract}

\section{INTRODUCTION}

In Continuum theory it is often useful to know more about special kinds of points in a continuum. A well known example is the classical result of Moore (see [Bo67, p. 177]) stating that every nondegenerate continuum must have at least two non-cut points (a non-cut point in a connected space is a point whose complement is connected); the result has been recently generalized to the shore points by Leonel ([Le13]) - precise definitions will be given later. We recommend to the reader the book of Nadler ([Na92]) as a general reference for many notions used throughout the paper.

More authors have investigated various properties of special sets in continua: Grace in [Gr81] provides a survey of results relating the notions of aposyndesis and weak cut point; Illanes in [Il01] shows that, in a dendroid, finite union of pairwise disjoint shore subdendroids is a shore set; among other results, a simple example of a planar dendroid in which the union of two disjoint closed shore sets is not a shore set is presented in [BMPV14]; in [Na07]

2010 Mathematics Subject Classification. 54F15, 54D10.

Key words and phrases. Continuum, shore point, non-cut point, arc-like continuum.

The work was supported by the grant GAČR 14-06989P. The third author is a junior researcher in the University Center for Mathematical Modeling, Applied Analysis and Computational Mathematics (Math MAC). 
Nall explores the relationship between center points and shore points in a dendroid; Illanes and Krupski study blockers and nonblockers for several kinds of continua ([IKr11]); and, using the results of [IKr11], Escobedo, López and Villanueva ([ELV12]) characterize some classes of locally connected continua - for further information on the subject see also [PV12, Le13].

Our aim is to study blocking properties of points in a general continuum. We laminate the set of non-cut points to six natural subsets (containing nonblock and shore points, among others) ordered by the inclusion and consider various questions related to them. Our interest is mainly focused on both the classes of arc-like and circle-like continua.

It is interesting to compare our lamination of non-cut points with several kinds of end points. The points of order one are points of colocal connectedness. In dendroids end points in the classical sense are exactly the points which are not weak cut points. In chainable continua the notion of end point is usually used in another sense and we show that in chainable continua the end points are closely related to non-block points.

The structure of our paper is as follows. In the next section we nominate the definitions of various kinds of non-cut points followed by illustrating examples. We recall several related results known from the literature and discuss the Borel hierarchy with respect to the notions in question. Also we show, generalizing the result from [Le13], that the sets of non-block points spans every nondegenerate continuum. Section 3 is devoted to the class of chainable (arc-like) continua. Among other results we show that any chainable continuum consisting of the non-block points is indecomposable - Corollary 3.6. In Section 4 we deal with the circle-like continua. The main result of this part states that every point in a circle-like continuum is a non-block point Theorem 4.5.

\section{LAMINATION OF NON-CUT POINTS}

We start by one illuminating example showing that the notion of a noncut point is relatively weak. Let $X$ be the continuum defined as the union of two $\sin \left(\frac{1}{x}\right)$-continua with the common vertical segment $S$. One can easily see that the set of non-cut points consists of all points in $S$ and two end points $e_{1}$ and $e_{2}$ of the sinusoidal branches. Choose $y \in S$ arbitrarily. The non-cut points $e_{1}, e_{2}$ do not have the same relationship to $X$ as the point $y$. There are arbitrarily small open neighborhoods of $e_{1}, e_{2}$ complements of which are connected, which is not true for $y$. The composant of $y$ is the whole $X$ whereas the composants of $e_{1}, e_{2}$ are proper subsets of $X$. The end points $e_{1}, e_{2}$ span $X$, i.e. no proper subcontinuum of $X$ contains all of them, at the same time the points in $S$ do not influence spanning of $X$ at all.

So it seems to be meaningful to distinguish various non-cut points in a continuum. Let us consider six kinds of non-cut points listed in Table 1. 


\begin{tabular}{|c|c|c|}
\hline notation & notion & definition \\
\hline P1 & $\begin{array}{l}x \text { is a point of } \\
\text { colocal connect- } \\
\text { edness }\end{array}$ & $\begin{array}{l}\text { there are arbitrary small open neigh- } \\
\text { borhoods of } x \text { complements of which are } \\
\text { connected }\end{array}$ \\
\hline P2 & $\begin{array}{l}x \text { is not a weak } \\
\text { cut point }\end{array}$ & $\begin{array}{l}\text { any pair of points distinct from } x \text { is con- } \\
\text { tained in a subcontinuum avoiding } x\end{array}$ \\
\hline P3 & $\begin{array}{l}x \text { is a non-block } \\
\text { point }\end{array}$ & $\begin{array}{l}\text { there exist subcontinua } A_{1} \subset A_{2} \subset \\
\cdots \subset X \text { such that } \bigcup_{n} A_{n} \text { is dense in } \\
X \backslash\{x\}\end{array}$ \\
\hline P4 & $\begin{array}{l}x \text { is a shore } \\
\text { point }\end{array}$ & $\begin{array}{l}\text { for each } \varepsilon>0 \text { there is an } \varepsilon \text {-dense sub- } \\
\text { continuum avoiding } x\end{array}$ \\
\hline P5 & $\begin{array}{l}x \text { is not a strong } \\
\text { center }\end{array}$ & $\begin{array}{l}\text { every pair of nonempty open sets is in- } \\
\text { tersected by a subcontinuum avoiding } \\
x\end{array}$ \\
\hline P6 & $\begin{array}{l}x \text { is a non-cut } \\
\text { point }\end{array}$ & the complement of $\{x\}$ is connected \\
\hline
\end{tabular}

TABLE 1.

It is easy to see that in general context any property with smaller number implies the one with greater number. On the other hand, as we show later, no property in Table 1 with greater number implies the one with smaller number.

Whyburn ([Wh39]) defined a continuum $X$ to be semi-locally connected at a point $x$ provided that if $U$ is an open subset of $X$ containing $x$, there is an open subset $V$ of $X$ lying in $U$ and containing $x$ such that $X \backslash V$ has a finite number of components. A continuum is semi-locally connected if it is semi-locally connected at every point.

A continuum $X$ is aposyndetic at a point $x$ provided that whenever $y$ is a point of $X$ distinct from $x$, there exists a subcontinuum $Y$ of $X$ and an open subset $U$ of $X$ such that $x \in U \subset Y \subset X \backslash\{y\}$. A continuum is aposyndetic if it is aposyndetic at every point.

REMARK 2.1. A continuum is semi-locally connected if and only if it is aposyndetic ([Ma05, Theorem 1.7.17]).

Using the results from [Wh39] we deduce that all the notions from Table 1 are equivalent.

Proposition 2.2. Let $X$ be a semi-locally connected continuum. Then all properties P1-P6 are equivalent. In particular, it is true when $X$ is locally connected.

Proof. It is sufficient to show that P6 implies P1. Let $x \in X$ be a noncut point. Choose an arbitrary open neighborhood $U$ of $x$. We assume that $X$ is semi-locally connected at $x$, so by definition there is an open neighborhood 
$V$ of $x$ such that $x \in V \subset U$ and some components $C_{1}, \ldots, C_{n}$ of $X \backslash V$ cover $X \backslash U$. By [Wh39, (6.2)] there exist subcontinua $X_{i j} \subset X \backslash\{x\}, 1 \leq$ $i, j \leq n$, such that $X_{i j}$ connects $C_{i}$ and $C_{j}$ for $i \neq j$. Obviously the set $W=U \backslash\left(\bigcup_{i} C_{i} \cup \bigcup_{i \neq j} X_{i j}\right)$ is an open neighborhood of $x$ satisfying $W \subset U$ and for which $X \backslash W$ is connected. Since every locally connected continuum is semi-locally connected ([Wh39, Example 2(i)]), the last part of our proposition follows.

Another natural notion for a point $x$ in a continuum $X$ which fits for our table could be:

P2': There exist subcontinua $A_{1} \subset A_{2} \subset \cdots \subset X$ such that

$$
X \backslash\{x\}=\bigcup_{n} A_{n} .
$$

Clearly P1 implies P2' which implies P2. However, it turns out that P2' only provides an alternative way of how to characterize the points with the property P2.

Proposition 2.3. Let $X$ be a continuum containing a point $x$. The following two properties are equivalent.

(i) $x$ has the property $P 2$ ( $x$ is not a weak cut point).

(ii) $x$ has the property $P 2^{\prime}$.

Proof. Clearly (ii) implies (i). In order to show the opposite implication, let $x \in X$ be not a weak cut point. Let $B(x, 1 / n)$ denote the open ball with the center at $x$ and the radius $1 / n$. Choose a point $p \in X \backslash\{x\}$ arbitrarily. Let $A_{n}$ be the connected component of $X \backslash B(x, 1 / n)$ containing $p$. Then for each sufficiently large $n$ the set $A_{n}$ is a continuum. We may assume that $A_{1} \neq \emptyset$. Then $\emptyset \neq A_{1} \subset A_{2} \subset \cdots$ and, since $x$ is not a weak cut point, $\bigcup_{n} A_{n}=X \backslash\{x\}$.

In order to complete the definitions from Table 1 we list several examples in which $\mathrm{P}(n+1)$ is not accompanied by $\mathrm{P} n$. For simplicity of notation, we write $\mathrm{P}(n+1) \backslash \mathrm{P} n$.

Example (P2 $\backslash \mathrm{P} 1)$. Let $X$ be a dendroid constructed as follows: if $P=$ $(0,0), Q=(1,0), A_{n}=(1+1 / n, 1 / n), B_{n}=(1+1 / n,-1 / n)$ and $C_{n}=$ $(0,-1 / n)$ are the points from $\mathbb{R}^{2}$, then the union of segments forms the desired dendroid

$$
X=P Q \cup \bigcup_{n}\left(P A_{n}\right) \cup\left(A_{n} B_{n}\right) \cup\left(B_{n} C_{n}\right) .
$$

The point $Q$ is neither a weak cut point nor a point of colocal connectedness.

ExAmPLE (P3 \P2). Any point of the vertical segment in the $\sin \left(\frac{1}{x}\right)$ continuum is a non-block point and a weak cut point. 
EXAmple (P4\P3). Let us denote by $C$ the Cantor middle third set, let $Y \subset \mathbb{R}^{2}$ be the union of all segments $[p, c]$ connecting the point $p=(0,1)$ with a point $c \in C \times\{0\}$. The continuum $Y$ is a special dendroid called the Cantor fan. Let $D_{n}=\left\{d_{1}^{n}, \ldots, d_{m(n)}^{n}\right\} \subset C \times\{0\}, n=1,2, \ldots$, be a finite $1 / n$-net in $C \times\{0\}$ such that $D_{i} \cap D_{j}=\emptyset$ for $i \neq j$. We define a decomposition $\sigma$ of $Y$ whose nondegenerate elements consist of finite sets

$$
\ell_{\alpha} \cap \bigcup_{i=1}^{m(n)}\left[p, d_{i}^{n}\right], n \in \mathbb{N}, \alpha \in[1-1 / n, 1),
$$

where $\ell_{\alpha}$ denotes the horizontal line of points with second coordinate $\alpha \in \mathbb{R}$. The quotient space $X=Y / \sigma$ is a continuum, since $\sigma$ is an upper semicontinuous decomposition. The continuum $X$ is a dendroid as well. The point $p$ is a shore point but not a non-block point.

EXAMPLE (P5\P4). With the above notation, let

$$
\left\{\left(a_{1}^{n}, a_{2}^{n}\right): a_{1}^{n} \neq a_{2}^{n} \text { for } n \in \mathbb{N} \text { and }\left\{a_{1}^{m}, a_{2}^{m}\right\} \cap\left\{a_{1}^{n}, a_{2}^{n}\right\}=\emptyset \text { for } m \neq n\right\}
$$

be dense in $C \times C$. We define a decomposition $\tau$ of the Cantor fan $Y$ whose nondegenerate elements consist of pairs of points

$$
\ell_{\alpha} \cap \bigcup_{i=1}^{2}\left[p, a_{i}^{n}\right], n \in \mathbb{N}, \alpha \in[1-1 / n, 1) .
$$

The quotient space $X=Y / \tau$ is again a dendroid. The point $p$ is neither a strong center nor a shore point.

EXAMPLE (P6 \P5). Any point of the common vertical segment of two $\sin \left(\frac{1}{x}\right)$-continua is a non-cut point and a strong center.

There are easy examples of continua without P2-points. For example, the closure of the graph of the function

$$
\sin \left(\frac{1}{1-|x|}\right), x \in(-1,1)
$$

has this property. In indecomposable continua there are no points of colocal connectedness (P1), even there are no points with property P2. On the other hand, every point of an indecomposable continuum is a non-block point (P3).

Let us briefly mention some results known from the literature related to the notions listed in our Table 1. In arcwise connected continua there are points of colocal connectedness (P1) ([KM79, Corollary 3.8]); the same is true for continua with exactly two arc components ([KM79, Corollary 3.11]). Every nondegenerate hereditarily decomposable continuum $X$ contains at least one subcontinuum $K$ with empty interior at which the continuum $X$ is colocally connected ([KM79, Corollary 3.5]). Hence any point of $K$ is a non-block point of $X(\mathrm{P} 3)$. In particular, every nondegenerate hereditarily decomposable 
continuum contains a non-block point. We show that every nondegenerate continuum contains at least two such points (Corollary 2.8). Recently, using the results of Bing $([\mathrm{Bi} 48])$, it has been proved that every nondegenerate continuum contains at least two shore points ([Le13]).

In what follows we concern the Borel types of sets of points listed in the table. We summarize our knowledge in the following.

Proposition 2.4. Let $X$ be a continuum. The following is true.

(i) The set of P1-points is of type $G_{\delta}$.

(ii) The set of P4-points is of type $G_{\delta}$.

(iii) The set of P5-points is of type $G_{\delta}$.

(iv) The set of P6-points is of type $F_{\sigma \delta}$.

Proof. (i) Let $C$ be the set of all points of colocal connectedness. For every $n \in \mathbb{N}$ there is an open cover $\mathcal{B}_{n}$ of $C$ by open sets of diameter less than $1 / n$ whose complements in $X$ are connected. It holds that $C=\bigcap_{n} \cup \mathcal{B}_{n}$.

(ii) For $n \in \mathbb{N}$ let $G_{n}$ be the set of all points $p$ in $X$ for which there exists a $(1 / n)$-dense continuum in $X \backslash p$. Then each $G_{n}$ is open and $\bigcap_{n=1}^{\infty} G_{n}$ is the set of all shore points in $X$.

(iii) Let $\mathcal{B}$ be a countable base of $X$. The set of all non-strong centers can be expressed as

$$
\bigcap_{A, B \in \mathcal{B}} \bigcup_{K}\{X \backslash K: K \cap A \neq \emptyset \neq K \cap B, K \text { is a continuum }\} .
$$

(iv) See [Wh42, Theorem 5.2].

We complete Proposition 2.4 by three examples.

ExAmple 2.5. (i) The set of P2-points need not be Borel. In a dendroid $X$, a point $x$ is an end point (in the classical sense) if whenever $x \in \gamma$ for some arc $\gamma \subset X$, then $x$ is an end point of $\gamma$. Obviously, the set of P2points in $X$ coincide with the set of all end points. The assertion follows from [NT90, Example 5], where the authors have found an example of a dendroid in which the set of all end points is co-analytic and not Borel.

(ii) The set of P $n$-points, $n \in\{1,4,5,6\}$, need not be of type $F_{\sigma}$. Let us denote by $X$ the Wazewski universal dendrite ([Wa23]), and by $E$ the set of all end points in $X$. As stated in the explanation of (i), the set $E$ coincides with the set of all P2-points. Since $X$ is locally connected, it follows from Proposition 2.2 that the sets of points with the properties P1-P6 coincide and hence they are equal to $E$. In particular, by Proposition 2.4(i) the set $E$ is of type $G_{\delta}$. It is known that $E$ is dense with an empty interior in $X$, hence by the Baire category theorem $E$ cannot be of type $F_{\sigma}$.

(iii) The set of all non-cut points is in general not of type $G_{\delta}$. We only sketch our argument. Let $Q=\left\{q_{n}: n \geq 0\right\}$ be the set of all rational numbers 
from the interval $(-1,1)$. Define the continuum $X$ as the closure of the graph of the function

$$
\sum_{n=0}^{\infty} \frac{1}{2^{n}} \sin \left(\frac{1}{x-q_{n}}\right), x \in(-1,1) \backslash Q .
$$

Let us denote by $N$ the set of all non-cut points in $X$. Obviously $(x, y) \in N$ if and only if $x \in Q \cup\{-1,1\}$, the set $N$ is dense and of the first category in $X$. By the Baire category theorem $N$ is not of type $G_{\delta}$.

It is of interest that the Borel complexity of the set of shore points is in general better than the one of the set of non-cut points. From this point of view the notion of a shore point is simpler than that of a non-cut point. Note that we still do not know the descriptive character of the set of non-block points. So we can pose the following.

QUESTION 2.1. Is the set of non-block points Borel? $5]$.

Our proof of Theorem 2.7 is based on the result of Bing [Bi48, Theorem

THEOREM 2.6. For each proper subset $R$ of the continuum $X$ there is a point $x$ of $X \backslash R$ such that the union of all continua that lie in $X \backslash\{x\}$ and intersect $R$ is dense in $X$.

We say that a subset $S$ of a continuum $X$ spans $X$ if no proper subcontinuum of $X$ contains $S$. The next theorem and its corollary generalize the fact that every nondegenerate continuum contains at least two non-cut points [Bo67, Le13].

TheOREm 2.7. Let $X$ be a continuum. Then the set of all non-block points spans $X$.

Proof. To the contrary, let $A$ be a proper subcontinuum of $X$ containing all non-block points. By Theorem 2.6 there exists a point $x \in X \backslash A$ such that the union of all continua that lie in $X \backslash\{x\}$ and intersect $A$ is dense in $X$. For some decreasing sequence $\left(\varepsilon_{n}\right)_{n=1}^{\infty}$ of positive reals converging to zero let us denote by $B_{n}$ the open ball with the center at $x$ and the radius $\varepsilon_{n}$; we can assume that $A \cap B_{1}=\emptyset$. For each $n$, let $A_{n}$ be the component of $X \backslash B_{n}$ containing $A$. Since $B_{n}$ is open, the set $A_{n}$ is a continuum. Moreover, $A_{n}$ is a subset of $A_{n+1}$ for $n=1,2, \ldots$ and any continuum $C \subset X \backslash\{x\}$ intersecting $A$ is a subset of $A_{n}$ for each sufficiently large $n$. Hence by Theorem 2.6 the union $\bigcup_{n=1}^{\infty} A_{n}$ is dense in $X \backslash\{x\}$, i.e. the point $x$ is a non-block point. Moreover, $x \notin A$ which is a contradiction.

Corollary 2.8. Let $X$ be a nondegenerate continuum. Then $X$ contains at least two non-block points. 


\section{Chainable continua}

In this section our attention will be focused on the class of chainable continua. For their own interest we state and prove several results describing various roles of distinct kinds of non-cut points from Table 1 . When arguing our statements we will repeatedly use the fact that chainable continua are hereditarily unicoherent ([Na92, Theorem 12.2]).

We start with two lemmas concerning the decomposability of a chainable continuum.

Lemma 3.1. Let $X$ be a chainable continuum such that $X=K \cup L$ for two proper subcontinua $K$ and $L$ of $X$. Then every point of $K \cap L$ is a strong center.

Proof. Let $p \in K \cap L$ and suppose that $p$ is not a strong center. Consider the nonempty open sets $X \backslash K$ and $X \backslash L$. Since $p$ is not a strong center, there is a continuum $M$ intersecting $K$ and $L$ but omitting $p$. It follows that

$$
M \cup(K \cap L)=(M \cap K) \cup(M \cap L) \cup(K \cap L)
$$

form a weak triod. This is a contradiction with the fact that chainable continua do not contain weak triods ([Na92, Corollary 12.7]).

Notice that the intersection $K \cap L$ from Lemma 3.1 can consist of the non-cut points only, see our Example P6 $\backslash P 5$ in Section 2.

A shore set in a continuum $X$ is a subset $A$ of $X$ such that, for each $\varepsilon>0$, there exists a subcontinuum $Y$ of $X$ such that the Hausdorff distance from $Y$ to $X$ is less than $\varepsilon$ and $A \cap Y=\emptyset$. In [Il01, Na07, BMPV14] the authors have studied in dendroids (or $\lambda$-dendroids) when the union of shore points (continua) is a shore set. In the case of chainable continua we deduce the following general result.

Proposition 3.2. The set of all shore points of a decomposable chainable continuum is a shore set.

Proof. Let $X$ be a decomposable chainable continuum and let $X=$ $K \cup L$ for two proper subcontinua $K$ and $L$ of $X$. By Theorem 2.7 there are non-block points hence also shore points $p \in K \backslash L$ and $q \in L \backslash K$. Related to $p, q$ there are sequences of continua $A_{n}$ and $B_{n}$ which converge to $X$ and such that $p \notin A_{n}$ and $q \notin B_{n}$. We may suppose that all $A_{n}$ and $B_{n}$ contain $K \cap L$. We define $M_{n}=\left(A_{n} \cap K\right) \cup\left(B_{n} \cap L\right)$. The sequence $M_{n}$ converges to $X$. We prove that the complement of $\bigcup M_{n}$ consists of all shore points. Clearly any point of $X \backslash \bigcup M_{n}$ is a shore point. On the other hand, suppose for contradiction that there is a shore point $r \in M_{n}$ for some $n \in \mathbb{N}$. Without loss of generality we may suppose that $r \in K$, notice that by Lemma 3.1 and Table 1 even $r \in K \backslash L$. Since $r$ is a shore point and $K \cap M_{n}$ is a proper closed subset of $K$, it follows that there is a subcontinuum $C$ of $X$ such that 
$C \cap\left(K \backslash M_{n}\right)$ is nonempty, $C \cap L=L \cap B_{n}$ and $r \notin C$. One can easily verify that $C \cup M_{n} \cup L$ is a weak triod in $X$ which is a contradiction. Thus the set of all shore points of $X$ is a shore set.

In a nondegenerate continuum $X$, a point $p \in X$ is a point of irreducibility provided that for some point $q \in X \backslash\{p\}$ no proper subcontinuum of $X$ contains $\{p, q\}$. Clearly $p$ is a point of irreducibility if and only if the composant of $p$ is a proper subset of $X$ (compare [Na92, Theorem 11.2]).

The next two lemmas hold true in the general context. They will be useful when proving Proposition 3.5 and Corollary 3.6. The first is from [Na92, Corollary 11.19]. The second generalizes [Le13, Theorem 3].

Lemma 3.3. Let $X$ be a nondegenerate continuum. The following two properties are equivalent.

(i) Every point $p \in X$ is a point of irreducibility of $X$.

(ii) $X$ is indecomposable.

LEMMA 3.4. Every point of irreducibility of a nondegenerate continuum $X$ is a non-block point.

Proof. If $p$ is a point of irreducibility, then for some point $q \in X \backslash\{p\}$ no proper subcontinuum of $X$ contains $\{p, q\}$. It means that the composant $\kappa(q)$ of $q$ does not contain the point $p$. Since the composant $\kappa(q)$ is dense in $X$ and can be expressed as a union of countably many proper subcontinua each of which contains $q$ ([Na92, Proposition 11.14]), the point $p$ is a non-block point.

The main statement of this section follows.

Proposition 3.5. Let $X$ be a chainable continuum and let $p \in X$. The following properties of $p$ are equivalent.

(i) $p$ is a point of irreducibility.

(ii) $p$ is a non-block point.

(iii) $p$ is a shore point.

(iv) $p$ is not a strong center.

Proof. By Lemma 3.4 (i) implies (ii). Moreover, (ii) implies (iii) and (iii) implies (iv) in general.

Let us prove (iv) implies (ii). If $X$ is an indecomposable continuum every point is a point of irreducibility ([Na92, Theorem 11.18]), so (iv) implies (i) and (i) implies (ii) by Lemma 3.4. Thus we can assume that $X$ is decomposable. Let $X=K \cup L$, where $K$ and $L$ are two proper subcontinua of $X$ and let $p$ be not a strong center of $X$. From Lemma 3.1 follows that $p \notin K \cap L$. Without loss of generality we may suppose that $p \in K \backslash L$. Let $\left\{B_{n}: n \in \mathbb{N}\right\}$ be the base of nonempty open subsets of $X \backslash\{p\}$. Since $p$ is not a strong center, there is for every $n \in \mathbb{N}$ a continuum $M_{n}$ intersecting $B_{n}$ and $X \backslash K$ 
such that $p \notin M_{n}$. It is enough to let $P_{n}=L \cup M_{1} \cup \ldots, \cup M_{n}$. We deduce that $P_{n}$ is a continuum not containing $p, P_{1} \subseteq P_{2} \subseteq \ldots$ and $P_{n}$ converge to $X$ in the Hausdorff metric. We have shown that $p$ is a non-block point, i.e. (iv) implies (ii).

It remains to prove that (ii) implies (i). In much the same way as above, let $p \in K \backslash L$. By Theorem 2.7 there is a non-block point $q \in L \backslash K$. We show that $X$ is irreducible between $p$ and $q$.

Suppose for contradiction that there is a proper subcontinuum $A$ of $X$ which contains both $p$ and $q$, let $x \in A \cap K \cap L ; x$ is a strong center by Lemma 3.1. At least one of the sets $K \backslash A, L \backslash A$ is nonempty. Assume the former possibility. Since $p$ is a non-block point, there is a sufficiently dense subcontinuum $B$ such that $p \notin B, x \in B$ and $(K \backslash A) \cap B$ is nonempty. It follows that $(L \cap A) \cup(K \cap A) \cup(K \cap B)$ forms a weak triod which is a contradiction.

Thus $X$ is irreducible between the points $p$ and $q$ and hence $p$ is a point of irreducibility.

Combining Proposition 3.5 and Lemma 3.3 we deduce the following.

Corollary 3.6. Let $X$ be a chainable continuum. The following properties are equivalent.

(i) Each point in $X$ is a non-block point.

(ii) $X$ is indecomposable.

Remark 3.7. By Proposition 3.5 the property P3 in Corollary 3.6 can be replaced by P4 or P5. Let $X$ be an arc of pseudoarcs ([Le85]). Then each point of $X$ is a non-cut point, at the same time $X$ is decomposable, hence Corollary 3.6 is not true for P6.

Let $X$ be a chainable continuum. A point $x \in X$ is called an end point of $X$ provided that for every $\varepsilon>0$ there is an $\varepsilon$-chain $B_{1}, \ldots, B_{n}$ covering $X$ such that $x \in B_{1}$. An end point in a chainable continuum need not fulfill the (classical) definition presented in Section 2 before Proposition 2.4. In [Do94] it has been shown that the cardinality of end points of a chainable continuum can be any cardinal number from $\left\{0,1, \ldots, \aleph_{0}, c\right\}$. In particular, it is known that the Buckethandle continuum is chainable and has exactly one end point ([Do08]). Gluing two Buckethandle continua together in their end points we find a chainable continuum with no end point.

There is a classical characterization of end points in chainable continua ([Do08, p. 32]). We recall two descriptions in the following statement.

Proposition 3.8. For a point $x$ of a nondegenerate chainable continuum $X$ the following conditions are equivalent.

(i) $x$ is an end point of $X$. 
(ii) Each nondegenerate subcontinuum of $X$ containing $x$ is irreducible between $x$ and some other point.

(iii) If there are two subcontinua of $X$ containing $x$, one of them contains the other.

From the above characterization of an end point in a chainable continuum and our Proposition 3.5 we conclude the following.

Proposition 3.9. Let $X$ be a chainable continuum and let $p \in X$. Then the following are equivalent.

(i) $p$ is an end point.

(ii) $p$ is a non-block point of every subcontinuum of $X$ which contains $p$.

Proof. It is a consequence of Propositions 3.8(i),(ii) and 3.5(i),(ii).

A point $x$ in a chainable continuum $X$ is called an absolute end point, provided that whenever $X$ is irreducible between $p$ and $q$, then either $x=p$ or $x=q$. By the definition there are at most two absolute end points in a chainable continuum. The notion of an absolute end point in chainable continua was introduced in [Ro88], where a number of equivalent characterizations was proved. We choose only the following one. A point $x$ is an absolute end point if and only if $x$ is a point of irreducibility and $X$ is locally connected at $x$ ([Ro88, Theorem 1.0]). We note that being locally connected at a point $x$ of a chainable continuum is the same as being connected im kleinen at $x$ ([Ro88, Theorem 1.7]).

It is easy to show that a point of order one in a chainable continuum is an absolute end point. The converse need not be true. For example the two end points of the arcless arc ([BPV13] ) are absolute end points but these are not of order one. This suggests to use the following notion. A continuum $X$ is said to be rim-connected at a point $x$ if there are arbitrarily small neighborhoods of $x$ whose boundaries are connected. From the Boundary bumping theorem we easily deduce that if a continuum $X$ is rim-connected at $x$, then $X$ is locally as well as colocally connected at $x$. We give two other characterizations of an absolute end point. One of them is based on Table 1 from Section 2, the other is using the notion of rim-connectedness. These results are using the following.

Lemma 3.10. Let $x$ be a point of irreducibility of a continuum $X$. Then the following are equivalent.

(i) $x$ is a point of local connectedness.

(ii) $x$ is a point of colocal connectedness.

Proof. (i) $\Longrightarrow$ (ii). Let $X$ be irreducible between $x$ and $y$. Let $U$ be any neighborhood of $x$. There is an open connected neighborhood $V$ of $x$ whose closure is a subset of $U$ and which avoids $y$. Let $K$ be the component of $X \backslash V$ which contains $y$. Clearly $K$ is a continuum intersecting the boundary of $V$ 
and hence $K \cup \operatorname{cl}(V)$ is a continuum. Since it contains $x$ and $y$, we conclude that it is equal to $X$. Hence $X \backslash K$ is a neighborhood of $x$ whose complement is connected.

(ii) $\Longrightarrow$ (i). Let $X$ be irreducible between $x$ and $y$. Let $U$ be any neighborhood of $x$. In $U$ there is an open neighborhood $V$ of $x$ avoiding $y$, whose complement is connected. Let us denote by $C$ the component of the point $x$ in $V$ and let us denote by $K$ the closure of $C$. Clearly $K$ is a continuum intersecting the boundary of $X \backslash V$. We get that $K \cup(X \backslash V)$ is a continuum containing $x$ and $y$ and thus it equals $X$. It follows that $K$ contains $V$ and thus $C=V$ is an open connected neighborhood of $x$ contained in $U$.

Proposition 3.11. The following are equivalent for a point $x$ in a chainable continuum $X$.

(i) $x$ is an absolute end point.

(ii) $x$ is a point of rim-connectedness.

(iii) $x$ is a point of colocal connectedness.

Proof. (i) $\Longrightarrow$ (ii). Let $x$ be an absolute end point. By [Ro88, Theorem 1.0] $x$ is a point of irreducibility at which $X$ is locally connected. Thus there is $y \in X$ such that $X$ is irreducible between $x$ and $y$. Let $U$ be any neighborhood of $x$ whose closure does not contain $y$. There is a connected open neighborhood $V \subseteq U$ of the point $x$. Let $K$ be the closure of $V$. Define $S$ to be the union of all subcontinua of $X \backslash K$ containing the point $y$. Let $L$ be the closure of $S$. First, we claim that $K \cap L \neq \emptyset$. Suppose to the contrary that this is not the case. Thus we can find an open set $W$ such that $L \subseteq W \subseteq \operatorname{cl}(W) \subseteq X \backslash K$. By the Boundary Bumping Theorem ([Na92, Theorem 5.4]) the component $C$ of the set $\operatorname{cl}(W)$ containing the point $y$ intersects the boundary of $W$. Since $C$ is disjoint with $K$ we get that $C \subseteq S$ and hence $C \subseteq L$. Thus $L$ intersects the boundary of $W$ which contradicts the fact that $L \subseteq W$ and $W$ is open.

It follows that $K \cup L$ is a continuum containing both $x$ and $y$ and thus $X=K \cup L$. Let $B$ be the boundary of $K$, we want to show that $B=K \cap L$. Clearly

$$
B=K \cap \operatorname{cl}(X \backslash K) \subseteq K \cap \operatorname{cl}(L)=K \cap L
$$

because $K \cup L=X$. For the opposite inclusion suppose that $z \in K \cap L$ is arbitrary. By the definition of $L$ there is a sequence of points $z_{n} \in S$ converging to $z$. Since $S$ is disjoint with $K$ it follows that $z \in B$.

By the unicoherence of $X$, the set $B=K \cap L$ is connected. Since the neighborhood $U$ was arbitrary we get that $x$ is a point of rim-connectedness.

(ii) $\Longrightarrow$ (iii). This implication holds in general.

(iii) $\Longrightarrow$ (i). Let $x$ be a point of colocal connectedness. In order to show that $x$ is an absolute end point it is enough to show that it is a point of irreducibility at which $X$ is locally connected ([Ro88, Theorem 1.0]). We have 
shown in Section 2 that $x$ is a non-block point (P1 implies P3) and thus it is a point of irreducibility by Proposition 3.5. Then from Lemma 3.10 we conclude that $x$ is a point of local connectedness of $X$ and hence $x$ is an absolute end point of $X$.

\section{Circle-like continua}

In this section we investigate the class of circle-like continua. The main tool of our approach will be the use of an inverse limit. Our main result is formulated in Theorem 4.5.

Let $\mathcal{P}$ be a collection of compact metric spaces. We say that a continuum $X$ is $\mathcal{P}$-like provided that for each $\varepsilon>0$ there is a continuous map $f$ from $X$ onto some member of $\mathcal{P}$ such that $\operatorname{diam} f^{-1}(f(x))<\varepsilon$ for each $x \in X$. In particular, if $\mathcal{P}$ consists of an arc (resp. a simple closed curve), then $X$ is called arc-like (resp. circle-like).

The next general result can be found for example in [Na92, Theorem 2.13].

Proposition 4.1. A continuum $X$ is $\mathcal{P}$-like if and only if $X$ is an inverse limit $\lim \left\{X_{i}, f_{i}\right\}$, where all the coordinate spaces $X_{i}$ are chosen from $\mathcal{P}$ and each bonding map $f_{i}: X_{i+1} \rightarrow X_{i}$ is continuous and onto.

It is known that the classes of arc-like and chainable continua coincide ([Na92, Theorem 12.11]). Some continua are both arc-like and circle-like, see for example the Buckethandle continuum ([Na92, 12.48]). In this section we deal with the circle-like continua from the point of view of our Table 1.

For a continuum $X$ and a continuous map $f: X \rightarrow X$ we say that $f$ is weakly confluent if for any subcontinuum $K \subset X$ there exists a component $L$ of $f^{-1}(K)$ such that $f(L)=K$.

Let $\mathbb{S}^{1}=\{z \in \mathbb{C}:|z|=1\}$. Consider a continuous map $f: \mathbb{S}^{1} \rightarrow \mathbb{S}^{1}$ of degree $\operatorname{deg}(f) \in \mathbb{Z}$. Let $F: \mathbb{R} \rightarrow \mathbb{R}$ be a lifting of $f$, i.e. the continuous map for which

$$
\varphi \circ F=f \circ \varphi \text { on } \mathbb{R},
$$

where $\varphi: \mathbb{R} \rightarrow \mathbb{S}^{1}$ is defined as $\varphi(x)=e^{2 \pi i x}$. Then

$$
F(x+1)=F(x)+\operatorname{deg}(f) \text { for each } x \in \mathbb{R} .
$$

In particular, if the degree $\operatorname{deg}(f)$ is nonzero the map $F$ is onto. Note that any map $F+m$ is also a lifting of $f$ for $m \in \mathbb{Z}$.

We start with one lemma providing an important ingredient of our next construction.

Lemma 4.2. (i) Any nonzero degree continuous self-map of the unit circle is weakly confluent.

(ii) Any continuous onto map $f: I \rightarrow J$, where $I, J$ are intervals, is weakly confluent. 
Proof. (i) Let $F: \mathbb{R} \rightarrow \mathbb{R}$ be a lifting of $f$. By our assumption on the degree, the map $F$ is onto.

Let $K$ be an arc in $\mathbb{S}^{1}$. Then $\varphi^{-1}(K)=[a, b]+\mathbb{Z}$ for some interval $[a, b] \subset \mathbb{R}, 0<b-a<1$. Since $F$ is continuous onto, there exist points $x, y \in \mathbb{R}$ such that $F(\{x, y\})=\{a, b\}$ and each point $t$ between $x, y$ is mapped by $F$ into $(a, b)$. Moreover, from (4.2) we conclude $|x-y|<1$. Let $J \subset \mathbb{R}$ be the interval with end points $x$ and $y$. Then $L^{\prime}=\varphi(J)$ is an arc in $\mathbb{S}^{1}$ and using (4.1) we deduce $f\left(L^{\prime}\right)=\varphi(F(J))=\varphi([a, b])=K$. So, if $L \subset \mathbb{S}^{1}$ is a component of $f^{-1}(K)$ containing $L^{\prime}$, then also $f(L)=K$ and $f$ is weakly confluent. (ii) We let the proof to the reader.

Let $X$ be a circle-like continuum. By Proposition 4.1 the continuum $X$ can be expressed as an inverse limit

$$
\lim _{\longleftarrow}\left\{\mathbb{S}^{1}, f_{i}\right\}=\left\{\left(\left(x_{i}\right)_{i=1}^{\infty}: f_{i}\left(x_{i+1}\right)=x_{i} \text { for each } i \in \mathbb{N}\right\} .\right.
$$

The space $X$ will be equipped with the metric

$$
d(x, y)=\sum_{i=1}^{\infty} \frac{\varrho\left(x_{i}, y_{i}\right)}{2^{i}}
$$

where $\varrho\left(x_{i}, y_{i}\right)$ denotes the Euclidean distance of $x_{i}, y_{i} \in \mathbb{S}^{1}$. For $n \in \mathbb{N}$ let $X_{n}=\left\{\left(x_{i}\right)_{i=1}^{n}:\left(x_{i}\right)_{i=1}^{\infty} \in X\right\}$ be a metric space endowed with the metric $d_{n}(x, y)=\sum_{i=1}^{n} \frac{\varrho\left(x_{i}, y_{i}\right)}{2^{i}}$. Let $\mathcal{H}_{d}$, resp. $\mathcal{H}_{d_{n}}$ be the induced Hausdorff metric on $X$, resp. $X_{n}$.

Lemma 4.3. Let each bonding map in (4.3) has nonzero degree. Fix a point $x=\left(x_{i}\right)_{i=1}^{\infty} \in X$. Then there is a countable set

$$
\left\{K_{i}^{j}: i \in \mathbb{N}, j \in\{1, \ldots, i\}\right\}
$$

of arcs in $\mathbb{S}^{1}$ satisfying (let $f_{j(i-1)}=f_{j} \circ \cdots \circ f_{i-1}$ for each $i>1$ and $1 \leq j<$ $\left.i-1, f_{(i-1)(i-1)}=f_{i-1}, f_{i(i-1)}=i d\right)$

(i) $K_{i}^{i} \subset \mathbb{S}^{1} \backslash\left\{x_{i}\right\}$ for $i \in \mathbb{N}$,

(ii) $f_{i}\left(K_{i+1}^{j}\right)=K_{i}^{j}$ for $i \in \mathbb{N}$ and $j \in\{1, \ldots, i\}$,

(iii) $K_{i}^{i} \supset K_{i}^{i-1} \cdots \supset K_{i}^{1}$ for $i \in \mathbb{N}$,

(iv) $f_{j i-1}\left(K_{i}^{i}\right) \supset K_{j}^{j}$ for each $i>1$ and $1 \leq j<i$,

(v) $\mathcal{H}_{d_{1}}\left(K_{1}^{1}, X_{1}\right)<1$,

(vi) For each $i>1$,

$$
\mathcal{H}_{d_{i}}\left(X_{i} \cap \prod_{j=1}^{i} f_{j(i-1)}\left(K_{i}^{i}\right), X_{i}\right)<1 / 2^{i-1} .
$$

PROOF. In the construction of $K_{i}^{j}$ we repeatedly use the fact that the bonding maps $f_{i}$ are continuous, onto and of a nonzero degree and apply 
Lemma 4.2. We proceed by the induction. In the $i$ th step, we choose arcs $K_{i}^{i-1}, K_{i}^{i-2}, \ldots, K_{i}^{1}, K_{i}^{i}$ (in written order):

STEP 1. We choose an $\operatorname{arc} K_{1}^{1} \subset \mathbb{S}^{1} \backslash\left\{x_{1}\right\}$ fulfilling the property (v).

SteP 2. With the help of Lemma $4.2(\mathrm{i})$ we choose an $\operatorname{arc} K_{2}^{1} \subset \mathbb{S}^{1}$ such that $f_{1}\left(K_{2}^{1}\right)=K_{1}^{1}$. Since the arc $K_{2}^{1}$ does not contain the point $x_{2}$ and $f_{1}\left(x_{2}\right)=x_{1}$, there exists an $\operatorname{arc} K_{2}^{2} \subset \mathbb{S}^{1} \backslash\left\{x_{2}\right\}$ such that $K_{2}^{2} \supset K_{2}^{1}$ (iii), $f_{1}\left(K_{2}^{2}\right) \supset K_{1}^{1}$ (iv) and (vi) is fulfilled for $i=2$.

SteP $i+1$. Let us assume that the $\operatorname{arcs} K_{i}^{i}, K_{i}^{i-1}, \ldots, K_{i}^{1}$ fulfilling (i)-(vi) have already been defined. Using Lemma 4.2 we can choose $\operatorname{arcs} K_{i+1}^{j}, j \in$ $\{1, \ldots, i\}$ satisfying (ii) and (iii). Since the arc $K_{i+1}^{i}$ does not contain the point $x_{i+1}\left(f_{i}\left(K_{i+1}^{i}\right)=K_{i}^{i}\right.$ and $x_{i} \notin K_{i}^{i}$ by (i)), there exists an arc $K_{i+1}^{i+1}$ (the length of which is sufficiently close to $2 \pi$ ) such that all the properties (i),(iii),(iv) and (vi) are satisfied.

This finishes our construction of the $\operatorname{arcs} K_{i}^{j}$ satisfying (i)-(vi).

For each $n \in \mathbb{N}$, let

$$
L_{i}^{n}=f_{i(n-1)}\left(K_{n}^{n}\right) \text { if } 1 \leq i<n, L_{i}^{n}=K_{i}^{n} \text { for } i \geq n .
$$

The key proposition follows.

Proposition 4.4. Let $X$ be a circle-like continuum such that each bonding map in (4.3) has nonzero degree.

(i) For each $n \in \mathbb{N}$, the set

$$
A_{n}=\lim _{\longleftarrow}\left\{L_{i}^{n}, f_{i}\right\}
$$

is a subcontinuum of $X$. Moreover, $A_{n} \subset A_{n+1}$.

(ii) $\bigcup_{n} A_{n} \subset X \backslash\{x\}$,

(iii) $\bigcup_{n} A_{n}$ is dense in $X$.

Proof. (i) By our definition of the arcs $L_{i}^{n}$ we conclude $f_{i}\left(L_{i+1}^{n}\right)=L_{i}^{n}$ for each $i \in \mathbb{N}$. Thus, the set $A_{n}$ is well defined for each $n \in \mathbb{N}$ and it is a subcontinuum in $X$. The inclusion $A_{n} \subset A_{n+1}$ directly follows from properties (iii) and (iv) of Lemma 4.3.

(ii) From property (i) of Lemma 4.3 we conclude $x_{n} \notin L_{n}^{n}=K_{n}^{n}$, hence $x \notin A_{n}$ for each $n$. It implies $x \notin \bigcup_{n} A_{n}$.

(iii) From the properties (v),(vi) of Lemma 4.3 we deduce

$$
\begin{aligned}
\mathcal{H}_{d}\left(A_{n}, X\right) & \leq \mathcal{H}_{d_{n}}\left(X_{n} \cap \prod_{j=1}^{n} f_{j(n-1)}\left(K_{n}^{n}\right), X_{n}\right)+\sum_{i=n+1}^{\infty} \frac{2}{2^{i}} \\
& <1 / 2^{n-1}+1 / 2^{n-1}=1 / 2^{n-2} .
\end{aligned}
$$


Using the above construction and the conclusion of Proposition 4.4 we conclude the following.

THEOREM 4.5. Let $X$ be a circle-like continuum. Then every point $x \in X$ is a non-block point.

Proof. If $X$ is also arc-like, then by [Bi62, p. 121] the continuum $X$ is indecomposable and the conclusion follows from Corollary 3.6. So in what follows we assume that $X$ is not arc-like. Then by [Ma05, Theorems 2.5.9$10]$ each bonding map in (4.3) can be assumed to have a positive degree and Proposition 4.4 can be applied.

We have proved that each point of a circle-like continuum has the property P3 from our Table 1. On the other hand, there are circle-like continua in which no point has the property $\mathrm{P} 2$, for example the circle of pseudoarcs is such a continuum ([BJ59]).

\section{REFERENCES}

[Bi48] R. H. Bing, Some characterizations of arcs and simple closed curves, Amer. J. Math. 70 (1948), 497-506.

[Bi62] R. H. Bing, Embedding circle-like continua in the plane, Canad. J. Math. 14 (1962), 113-128.

[BJ59] R.H. Bing and F.B. Jones, Another homogeneous plane continuum, Trans. Amer. Math. Soc. 90 (1959), 171-192.

[BMPV14] J. Bobok, R. Marciňa, P. Pyrih and B. Vejnar, Union of shore sets in a dendroid, Topology Appl. 161 (2014), 206-214.

[BPV13] J. Bobok, P. Pyrih and B. Vejnar, Half-homogeneous chainable continua with end points, Topology Appl. 160 (2013), 1066-1073.

[Bo67] K. Borsuk, Theory of retracts, Polish Scientific Publishers, Warsaw, 1967.

[Do94] J. Doucet, Cardinality, completeness, and decomposability of sets of endpoints of chainable continua, Topology Appl. 60 (1994), 41-59.

[Do08] J. Doucet, Sets of endpoints of chainable continua, Topology Proc. 32 (2008), $31-35$.

[Gr81] E. E. Grace, Aposyndesis and weak cutting, in: General topology and modern analysis, Academic Press, New York, 1981, 71-82.

[ELV12] R. Escobedo, M. de Jesús López and H. Villanueva, Nonblockers in hyperspaces, Topology Appl. 159 (2012), 3614-3618.

[Il01] A. Illanes, Finite unions of shore sets, Rend. Circ. Mat. Palermo (2) 50 (2001), 483-498.

[IKr11] A. Illanes and P. Krupski, Blockers in hyperspaces, Topology Appl. 158 (2011), $653-659$.

[KM79] J. Krasinkiewicz and P. Minc, Continua and their open subsets with connected complements, Fund. Math. 102 (1979), 129-136.

[Le13] R. Leonel, Shore points of a continuum, Topology Appl. 161 (2014), 433-441.

[Le85] W. Lewis, Continuous curves of pseudo-arcs, Houston J. Math. 11 (1985), 9199.

[Ma05] S. Macias, Topics on continua, Chapman and Hall/CRC, Boca Raton, 2005.

[Na92] S. B. Nadler, Continuum theory. An introduction, Marcel Dekker, New York, 1992. 
[Na07] V. C. Nall, Centers and shore points of a dendroid, Topology Appl. 154 (2007), 2167-2172.

[NT90] J. Nikiel and E. D. Tymchatyn, Sets of end-points and ramification points in dendroids, Fund. Math. 138 (1991), 139-146.

[PV12] P. Pyrih and B. Vejnar, A lambda-dendroid with two shore points whose union is not a shore set, Topology Appl. 159 (2012), 69-74.

[Ro88] I. Rosenholtz, Absolute endpoints of chainable continua, Proc. Amer. Math. Soc. 103 (1988), 1305-1314.

[Wa23] T. Wazewski, Sur un continu singulier, Fundamenta Mathematicae 4 (1923), 214-245.

[Wh39] G. T. Whyburn, Semi-locally connected sets, Amer. J. Math. 61 (1939), 733-749.

[Wh42] G. T. Whyburn, Analytic topology, American Mathematical Society, New York, 1942.

J. Bobok

Faculty of Civil Engineering

Czech Technical University in Prague

P. Pyrih

Faculty of Mathematics and Physics

Charles University in Prague

11800 Prague

Czech Republic

B. Vejnar

Faculty of Mathematics and Physics

Charles University in Prague

11800 Prague

Czech Republic

E-mail: vejnar@karlin.mff.cuni.cz

Received: 14.8.2014.

Revised: 18.2.2015. 